\title{
Finite Element Method Analysis of Microfluidic Channel with Integrated Dielectrophoresis Electrodes for Biological Cell Permeabilization and Manipulation
}

\author{
V. Novickij ${ }^{1,2}$, A. Grainys ${ }^{1,2}$, J. Novickij ${ }^{1,2}$ \\ ${ }^{1}$ Department of Electrical Engineering, Faculty of Electronics, Vilnius Gediminas Technical University, \\ Naugarduko street 41, Vilnius LT-03327, Lithuania, e-mail: vitalij.novickij@vgtu.It \\ ${ }^{2}$ High Magnetic Field Laboratory, Faculty of Electronics, Vilnius Gediminas Technical University, \\ Naugarduko street 41, Vilnius LT-03327, Lithuania, e-mail: smll@vgtu.lt
}

\begin{abstract}
The microfluidic channel with a planar inductive microcoil for the cell membrane permeabilization and the integrated planar electrodes for cell dielectrophoretic manipulation is proposed and analyzed in the study. The analyzed setup is based on the dielectrophoretic entrapment of the biological cell followed by membrane permeabilization using high pulsed magnetic field. The finite element method analysis of the DEP force and the generated pulsed magnetic field is performed. Based on finite element method analysis the potential applications of the setup in the fields of drug delivery, biomedicine and biotechnology are discussed.
\end{abstract}

Keywords: Pulsed magnetic field, dielectrophoretic entrapment, membrane permeabilization, biological cells, drug delivery

\section{INTRODUCTION}

$\mathrm{B}$ IOELECTRONICS has become one of the most rapidly developed scientific fields in the recent years because of high demand of new techniques and methods in the area of biomedicine, bioengineering, biotechnology and ecology [1]. One of the most popular techniques for treatment and manipulation of biological cells are dielectrophoresis (DEP) and electroporation. DEP is a technique based on polarization when alternated nonuniform electric field is used and could be successfully applied to manipulation of any polarizable particles ranging from $\mathrm{nm}$ to $\mu \mathrm{m}$ range [2]. Traditionally DEP systems are implemented using standard setup of microfluidic channel with metal electrodes on the bottom which are in direct contact with the cells [3]. Electroporation is used to permeabilize the thin dielectric membrane of the cells and allow transportation of chemicals inside and outside the cell, however, pulsed high power treatment with electric field is required [4]. Microfluidic systems implementing both these techniques together are used very rarely because of the difference in the electrode structures of these two techniques and complexity of fabrication, nevertheless, there is a demand of such microfluidic systems. Also there is a risk of electrical breakdown between the electrodes due to technique dependence on the conductivity of the medium. However, preliminary studies have shown that high microsecond magnetic fields allow achieving high efficiency rates of cell membrane permeabilization and application of pulsed magnetic field in this area of biomedical research allows achieving contactless treatment of biological objects and major simplification of the whole system. Also implementation of both techniques on a single microfluidic chip would allow non-direct measurement of the treatment intensity. The work presented is focused on the finite element method analysis of the proposed structure of the microfluidic chip for pulsed magnetic field treatment of biological cells. The aim is to develop a structure that could be successfully integrated in the lab on chip devices, microfluidic channels and other separation and particle manipulation devices that require a controlled process of cell membrane permeabilization. Determination of the parameters and optimal structure of the microfluidic system based on high magnetic fields and DEP cells entrapment module would allow creating a new type of micro scale manipulation devices that could be successfully applied in the field of bioengineering and bioelectronics.

\section{SUBJECT \& METHODS}

\section{A. Dielectrophoresis and the biological cell}

Traditionally the biological cell models are analyzed as a conducting sphere with a thin $5-50 \mathrm{~nm}$ dielectric layer representing the membrane [5]. The biological cell model is presented in Fig.1.

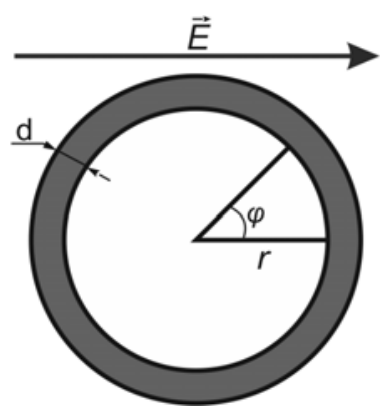

Fig.1. The biological cell model.

It should be noted that the membrane thickness $d$ is in the range of $5-50 \mathrm{~nm}$, while the cell radius $r$ can vary in the range from several to hundreds of $\mu \mathrm{m}$ depending on the type of the cell [6]. The sphere consists of an isotropic and homogeneous dielectric, therefore, when the external electric field $E$ is applied, the dielectric layer will be homogeneously polarized and the internal $E_{i}$ electric field 
symmetric about the $\mathrm{x}$ - axis will be created, which results in the occurrence of particle potential $\psi_{p}[7]$ :

$$
\psi_{p}=-\boldsymbol{E}_{i} x
$$

where, $x=r \cos \theta$ in polar coordinates. Such polarization could be interpreted as an induced dipole of an effective moment $m$ located in the particle center [7]:

$$
m=\rho v \boldsymbol{E}=4 \pi \varepsilon_{m} r^{3} \boldsymbol{E}\left[\frac{\varepsilon_{p}^{*}-\varepsilon_{m}^{*}}{\varepsilon_{p}^{*}+2 \varepsilon_{m}^{*}}\right],
$$

where $\rho$ is the effective polarizability of the particle, $v$ is the volume, $\varepsilon_{m}$ is the absolute permittivity of the medium, $\varepsilon_{p}^{*}, \varepsilon_{m}^{*}$ are the relative permittivities of the particle and the medium, respectively.

Subsequently, the dielectrophoretic force the particle is experiencing in the non-uniform electric field could be defined as [8]:

$$
\begin{aligned}
& \boldsymbol{F}=\rho v(\boldsymbol{E} . \nabla) \boldsymbol{E}=4 \pi \varepsilon_{m} r^{3} \boldsymbol{E}\left[\frac{\varepsilon_{p}^{*}-\varepsilon_{m}^{*}}{\varepsilon_{p}^{*}+2 \varepsilon_{m}^{*}}\right](\boldsymbol{E} . \nabla) \boldsymbol{E}= \\
& =2 \pi \varepsilon_{m} r^{3}\left[\frac{\varepsilon_{p}^{*}-\varepsilon_{m}^{*}}{\varepsilon_{p}^{*}+2 \varepsilon_{m}^{*}}\right]\left(\nabla \boldsymbol{E}^{2}\right) .
\end{aligned}
$$

As it can be seen in (3) the DEP force is proportional to the gradient of the square of the electric field. Also a transition from one type of DEP to another may occur when the frequency of the applied electric field is being altered. The frequency when the transition occurs is the crossover frequency and is defined as [8]:

$$
f_{x 0}=\frac{1}{2 \pi \varepsilon_{0}} \sqrt{\frac{\left(\sigma_{m}-\sigma_{p}\right)\left(\sigma_{p}-2 \sigma_{m}\right)}{\left(\varepsilon_{p}-\varepsilon_{m}\right)\left(\varepsilon_{p}-2 \varepsilon_{m}\right)}} .
$$

Depending on the frequency, the particles are either repelled from the field exciter (negative DEP) or attracted towards the field source (positive DEP). The aim of this study is to develop a cell entrapment system, therefore, negative DEP will be used.

\section{B. Cell membrane permeabilization}

Electroporation is a phenomenon based on permeabilization of the cell membrane during pulsed treatment when the transmembrane potential of the cell is being altered. The transmembrane potential could be defined as [9]:

$$
V_{T M}=\frac{3 r}{2} \boldsymbol{E} \cos \theta,
$$

where $r$ is the radius of the cell and $\boldsymbol{E}$ is the electric field applied. When the potential is high enough the membrane of the cell becomes temporarily permeable, which is widely used in drug delivery technologies. In this study pulsed magnetic field will be used for membrane permeabilization of biological cells. The dependence of the induced electric field on the alternating magnetic field could be found from the Maxwell-Faraday equation:

$$
\nabla \times \boldsymbol{E}=\frac{-\partial \boldsymbol{B}}{\partial t},
$$

where $\boldsymbol{B}$ is magnetic flux density and $t$ is time. Based on the empirical studies in this area, microsecond range magnetic field pulses with magnetic flux density in the range of 4-5 T and rise times in the range of $1-2 \mu \mathrm{s}$ are required to induce high enough electric field to successfully permeabilize the cell membrane [10]. Therefore, during analysis of the magnetic field generated by a planar coil, magnetic flux density of at least 4-5 $\mathrm{T}$ needs to be achieved. The arrangement of windings in a typical planar coil is shown in Fig.2.

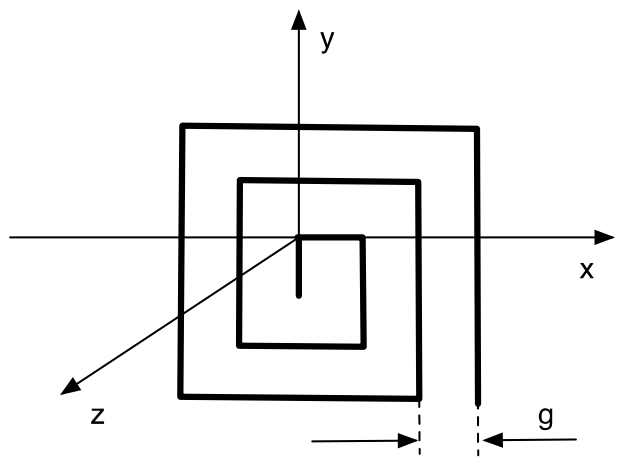

Fig.2. The arrangement of windings in a typical planar coil.

It can be seen in Fig.2. that the four wire segments form a spiral. Thus, in order to determine the magnetic field distribution of the coil the superposition of the magnetic fields of each wire needs to be evaluated. Magnetic flux density could be evaluated by applying Biot-Savart's law to each wire segment [11]:

$$
\begin{aligned}
& B_{X}=0, \\
& B_{Y}=-\frac{z I \mu_{0}}{4 \pi\left[(a-y)^{2}+z^{2}\right]}\left[\begin{array}{c}
\frac{L-x}{\sqrt{(L-x)^{2}}+(a-y)^{2}+z^{2}}+ \\
\left.+\frac{L+x}{\sqrt{(L+x)^{2}}+(a-y)^{2}+z^{2}}\right]
\end{array},\right. \\
& \left.B_{Z}=-\frac{(a-y) I \mu_{0}}{4 \pi\left[(a-y)^{2}+z^{2}\right.}\right]\left[\begin{array}{l}
\frac{L-x}{\sqrt{(L-x)^{2}}+(a-y)^{2}+z^{2}}+ \\
+\frac{L+x}{\sqrt{(L+x)^{2}}+(a-y)^{2}+z^{2}}
\end{array}\right],
\end{aligned}
$$

where $x, y, z$ are the Cartesian coordinates of the point where the magnetic field is calculated, $I$ is the current, $a$ is the 
distance of the wire from the $x-z$ plane, $L$ is the half length of the wire segment and $\mu_{0}$ is the magnetic constant. Any disposition of the wire segment can be recalculated by rotation or translation of the system. If the magnetic field is calculated near the conductor the expression could be further simplified and take the following form [12]:

$$
B_{Z}=-\frac{I \mu_{0}}{4 \pi y}\left[\begin{array}{l}
\frac{L-x}{\sqrt{\left(x^{2}+y^{2}+L^{2}-2 L x\right)^{3}}}+\text {. } \\
+\sqrt{\left(x^{2}+y^{2}+L^{2}-2 L x\right)^{3}}
\end{array}\right] \text {. }
$$

As it can be seen in $(7-8)$ the magnetic flux density depends on the current value. Application of high currents in microfluidic chips is not convenient due to generated heat, therefore, a balance between optimal current value and the resultant magnetic field value must be found [13].

\section{DEP entrapment module}

The proposed microfluidic system is composed of DEP and cell membrane permeabilization modules, which have to be analyzed separately. The purpose of the DEP electrodes is to entrap the cells in a finite volume until the cell permeabilization is performed. For such task a quadruple electrode structure is proposed and analyzed using FEM. The structure of the DEP electrodes is shown in Fig.3. Each pair of the electrodes is connected to an AC voltage source and $7 \mathrm{~V}_{\mathrm{p}-\mathrm{p}}$ is supplied. The width and the height of the simulated microchannel are 400 and $50 \mu \mathrm{m}$, respectively.

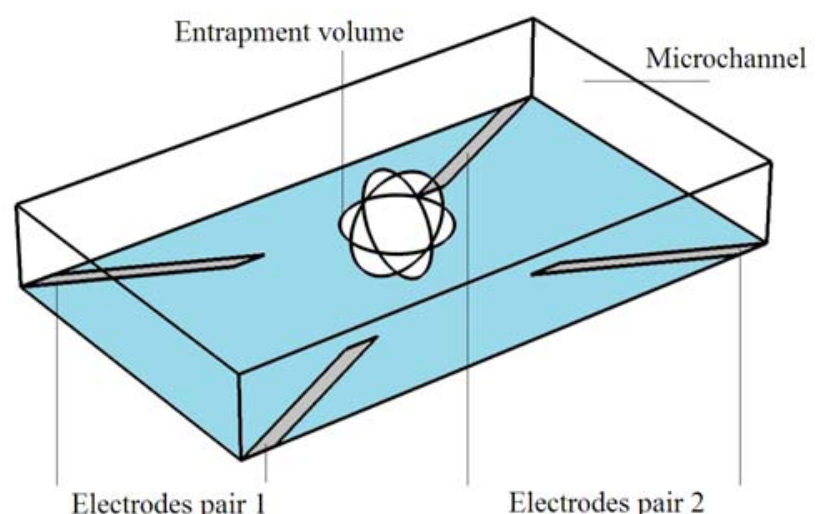

Fig. 3. The structure of the DEP electrodes.

According to (3) the DEP force is proportional to the gradient of the square of the electric field. According to (8) this parameter must be higher than $10^{12} \mathrm{~V}^{2} \mathrm{~m}^{-3}$ to exert sufficient force of $5-50 \mathrm{pN}$ to manipulate cells from small to medium size. The distribution of the gradient of the square of the electric field in the proposed microfluidic channel structure is shown in Fig.4.

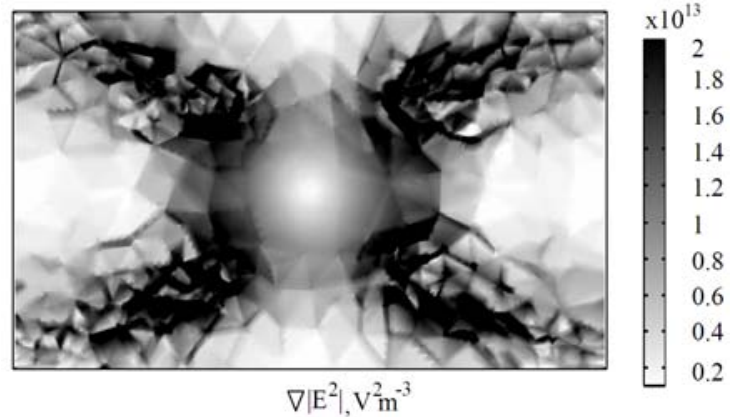

Fig.4. Distribution of the gradient of the square of the electric field in the proposed microfluidic channel.

The $\nabla E^{2}$ value is sufficient for the entrapment of the biological cells inside the finite volume until the cell membrane permeabilization is performed. It should be noted that the DEP system is calibrated based on the electrical properties of the cells before magnetic field treatment. The frequency of electric field needs to be altered to achieve the best Clausius-Mossotti factor for each cell type. The parameters that have been used in the simulation are summarized in Table 1.

Table 1. The parameters that have been used and evaluated in the simulation.

\begin{tabular}{|c|c|c|}
\hline Parameter & Value & Requirement \\
\hline Channel width & $400 \mu \mathrm{m}$ & $>300 \mu \mathrm{m}$ \\
\hline Channel height & $50 \mu \mathrm{m}$ & $>30 \mu \mathrm{m}$ \\
\hline$\nabla E^{2}$ & $>1 \times 10^{13}$ & $>1 \times 10^{12}$ \\
\hline Electrodes gap & $100 \mu \mathrm{m}$ & $>50 \mu \mathrm{m}$ \\
\hline Voltage & $7 \mathrm{~V}_{\mathrm{p}-\mathrm{p}}$ & $<10 \mathrm{~V}_{\mathrm{p}-\mathrm{p}}$ \\
\hline Cell size & $>2 \mu \mathrm{m}$ & $>2 \mu \mathrm{m}$ \\
\hline
\end{tabular}

\section{Microcoil}

The cell membrane permeabilization module consists of a planar microcoil and a pulsed power generator. As it was mentioned above the planar microcoil should be generating fields up to 4-5 $\mathrm{T}$ in a finite volume, where the cells are entrapped by DEP. The resultant structure of the planar microcoil integrated in the microfluidic channel is shown in Fig.5.

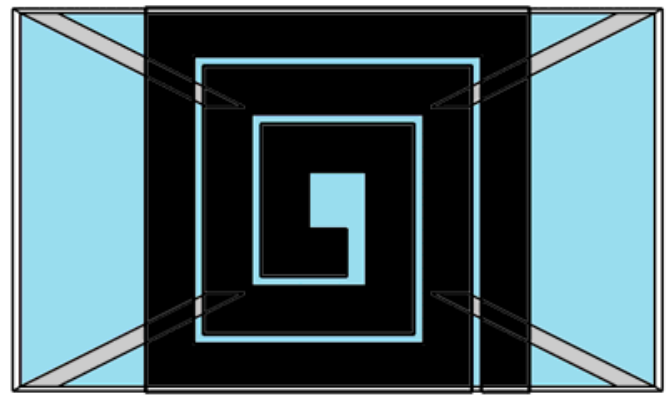

Fig.5. The resultant structure of the planar microcoil integrated in the microfluidic channel. 
The coil is positioned on the top of the microfluidic channel directly in the middle of the cell entrapment area. It should be noted that the dimensions of the coil and the number of windings could be increased with an increase of the microfluidic channel. The whole structure is scalable, however, the change in current value will be required. In the simulation the peak current supplied is $220 \mathrm{~A}$. The resultant magnetic flux density distribution inside the microfluidic channel is shown in Fig.6.

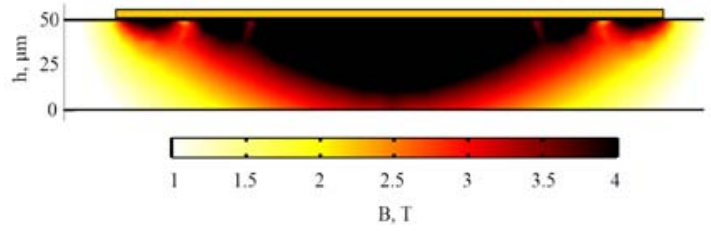

Fig.6. The resultant magnetic flux density distribution inside the microfluidic channel.

The magnetic flux density in the $95 \%$ of the cell entrapment volume is higher or equal to $4 \mathrm{~T}$. The microcoil does not have any galvanic contact with the medium or the DEP electrodes. However, fabrication of structures on the two walls of microfluidic channel is not always possible. Therefore, the planar coil structure could be positioned at the same plane as DEP electrodes, however, a thin micrometer range dielectric layer will be required.

The control schematic of the proposed setup implementation is shown in Fig.7.

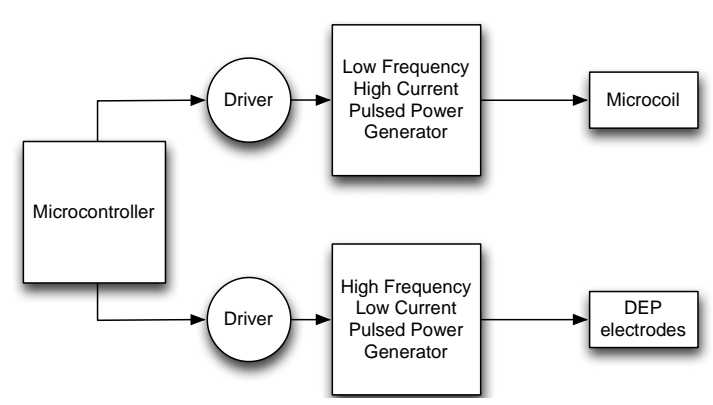

Fig.7. The schematic of the proposed setup implementation.

As it can be seen in Fig.7. two pulsed generators controlled by a single microcontroller unit are proposed. Such implementation is recommended when high current is required, which implies that high frequency is hardly achievable. Therefore, one generator works in low frequency high current mode for high magnetic field generation and the other in high frequency low current mode for DEP entrapment of the specimens. However, if high frequency semiconductor switches are applied, it is possible to develop a single generator for both DEP and magnetic field generation.

\section{RESULTS}

A microfluidic chip based on dielectrophoresis and pulsed magnetic field treatment for cell membrane permeabilization has been proposed and analyzed using the finite element method analysis. Based on the simulation results it was shown that it is possible to fabricate an innovative microfluidic channel, where biological cells are subjected to pulsed high magnetic fields up to 4-5 $\mathrm{T}$ and are manipulated by DEP. Implementation of the proposed setup would allow affecting the biological cells uniformly. Also it is presumed that after pulsed magnetic field treatment when the electrical properties of the cells change, the DEP force affecting the specimens will be different for magnetoporated and nonmagnetoporated cells. Therefore, by alternating the frequency of the electric field, the threshold when the cells start to leave the entrapment area could be used as a nondirect measurement for estimation of the treatment intensity.

\section{DISCUSSION / CONCLUSIONS}

The proposed microfluidic system consists of two main modules. The dielectrophoresis module is used to entrap the biological cells. The second module is the microcoil for cell membrane permeabilization. High current microsecond pulses in the range of $200 \mathrm{~A}$ are used for magnetic field generation. It is possible to develop a pulsed power generator that will be applicable for both DEP and magnetic field generation, which will result in simplification of the facility. The proposed setup could have high applicability in the area of bioelectronics and lab on chip devices, because of implementation of pulsed magnetic field permeabilization module, which eliminates the electrical breakdown, electrode contamination and medium heating problems that are present in classical electroporation cuvettes.

\section{REFERENCES}

[1] Sarpeshkar, R. (2010). Ultra Low Power Bioelectronics: Fundamentals, Biomedical Applications, and Bio-Inspired Systems. Cambridge University Press.

[2] Pohl, H.A. (1978). Dielectrophoresis: The Behavior of Neutral Matter in Nonuniform Electric Fields. Cambridge University Press.

[3] Lei, U., Lo, Y.J. (2012). Review of the theory of generalised dielectrophoresis. IET Nanobiotechnology, 5 (3), 86-106.

[4] Cahill, K. (2010). Cell - penetrating peptides, electroporation and drug delivery. IET Systems Biology, 4 (6), 367-378.

[5] Chenguo, Y., Dengbin, M., Chengxiang, L., Caixin, S., Yan, M. (2007). Study of transmembrane potentials of inner and outer membranes induced by pulsed-electricfield model and simulation. IEEE Transactions on Plasma Science, 35 (5), 1541-1549.

[6] EL-Hag, A., Jayaram, S.H. (2008). Effect of biological cell size and shape on killing efficiency of pulsed electric field. In IEEE International Conference on Dielectric Liquids (ICDL 2008), 30 June - 3 July 2008. IEEE, 1-4.

[7] Pethig, R. (2007). Cell physiometry tools based on dielectrophoresis. In Micro/Nano Technology for Genomics and Proteomics. Springer.

[8] Holzel, R., Bier, F.F. (2003). Dielectrophoretic manipulation of DNA. IEE Proceedings Nanobiotechnology, 150 (2), 47-53. 
[9] Ivorra, A. (2010). Tissue electroporation as a bioelectric phenomenon: Basic concepts. In Irreversible Electroporation. Springer.

[10] Grainys, A. et. al. (2012). High power facilities for electroporation of biological cells in pulsed magnetic fields. In 19th International Conference on Microwave Radar and Wireless Communications (MIKON), 21-23 May 2012. IEEE, Vol. 2, 508-511.

[11] Garcia, A. et. al. (2001). A method for calculation the magnetic field produced by a coil of any shape. Sensors and Actuators A, 91, 230-232.
[12] Frollo, I., Andris, P., Pribil, J., Vojtisek, L., Dermek, T., Valkovic, L. (2010). Measurement and imaging of planar electromagnetic phantoms based on NMR imaging methods. Measurement Science Review, $10(3), 97-101$.

[13] Bartkevičius, S., Novickij, J. (2008). The influence of pulsed magnet heating on maximal value of generated magnetic field. Measurement Science Review, 8 (4), 94-97.

Received January 8, 2013. Accepted June 10, 2013. 